\title{
Prediction Experiments for Reliability of Machine Tool by Product Performance Data
}

\author{
Xintao Xia ${ }^{\mathrm{a}}$, Wenhuan Zhu ${ }^{\mathrm{b}^{*}}$ and Zhen Chang ${ }^{\mathrm{c}}$ \\ School of Mechatronical Engineering, Henan University of Science and Technology, Luoyang \\ 471003, China

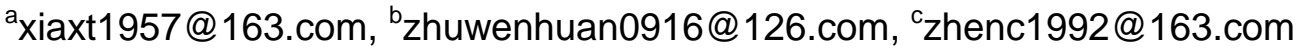

Keywords: Prediction; Reliability; Machine tool; Measurement uncertainty; Fuzzy set theory.

\begin{abstract}
For processing mass high quality products, it is crucial that the machine tool can keep a good running in the manufacturing process. In order that the product percent of pass is up to the standard of production, it needs to predict the reliability of the machine tool as soon as possible, which belongs to the probability distribution unknown. Based on the fuzzy set theory, prediction experiments for the reliability of the machine tool by product performance data can be realized. Prediction experiment of judging the reliability of the machine tool shows that under the confidence level $P=95.44 \%$, if the relative error of the measurement uncertainty of the experimental data is not more than $45 \%$, the machine tool is reliable; otherwise, the machine tool is not reliable. Prediction experiment of evaluating the reliability of the machine tool shows that it can be accurately predicted the reliability of the machine tool according to the fuzzy set theory.
\end{abstract}

\section{Introduction}

In the mechanical industry, the machine tool is used to manufacture the components of the complex products such as aircrafts, steamships, high speed trains etc. With the market competition increasingly fierce of mechanical products, more and more researchers study the reliability of the machine tool to reach the high performance indicator of mechanical products[1]. Obviously, it is necessary to do experiment to predict the reliability of the machine tool.

For the normal machine tool, the fluctuation range of the product performance data in the manufacturing process owns minor variations in large or small. If the variation range is still in the allowed range of processing, and the machine tool in this stage is considered as reliable. As the accumulation of processing time, the fluctuation range of the product performance data is larger and larger to a certain extent in which it can be considered that the manufacturing process is unreliable[2-6].

According to the fuzzy set theory[7-10], the product performance data under investigation are processed to predict accurately the reliability of the machine tool using the evaluation method[7] in this paper.

\section{Prediction experiments for the reliability of the machine tool}

Prediction experiment of judging the reliability of the machine tool. Based on the evaluation method[7], in the simulation experiment, the simulation data are generated by computer simulation, which can be used to simulate the measured data of the product performance. The simulation data can reflect the change degree of the reliability of the machine tool which is from reliable to unreliable.

10 simulation data which obey normal distribution with the mathematical expectation $E_{1}=0$ and the standard deviation $\sigma=0.01$ are generated by Monte Carlo method, are considered as the 10 measured data of the product performance, constituting the intrinsic sequence $X_{1}$ of the machine tool.

The expanded uncertainty $\left( \pm 2 \sigma_{1}\right)$ of the intrinsic sequence $X_{1}$ is defined as the allowed fluctuation 
range of the product performance data. The simulation data are generated and their fluctuation ranges are assumed from $\pm 2 \sigma_{1}$ to $\pm 3 \sigma_{1}$. According to the statistics, it can be considered that the machine tool is not reliable if the fluctuation range of the simulation data increases to $\pm 3 \sigma_{1}$. Let $3 \sigma_{1}=2 \sigma_{6}, 5$ groups of 10 simulation data are generated by Monte Carlo method, respectively, and considered as the evaluated sequences $X_{2}, X_{3}, X_{4}, X_{5}, X_{6}$, respectively, which obey normal distributions with the mathematical expectations $E_{2}=E_{3}=E_{4}=E_{5}=E_{6}=0$ and the standard deviations $\left\{\sigma_{2}, \sigma_{3}, \sigma_{4}, \sigma_{5}, \sigma_{6}\right\}=\{0.011,0.012,0.013,0.014,0.015\}$, respectively.

Thus, 6 simulation data sequences are obtained by Monte Carlo method for judging the reliability of the machine tool $(M=6)$. Simulation results are shown in Table 1 and Table 2 and all of simulation data can constitute the simulation data sequence $X_{60}$, as shown in Fig. 1.

Table 1 Simulation data sequences obeying normal distributions with different standard deviations

\begin{tabular}{ccccccc}
\hline$\sigma$ & $\sigma_{1}=0.010$ & $\sigma_{2}=0.011$ & $\sigma_{3}=0.012$ & $\sigma_{4}=0.013$ & $\sigma_{5}=0.014$ & $\sigma_{6}=0.015$ \\
\hline$X_{m}$ & $X_{1}$ & $X_{2}$ & $X_{3}$ & $X_{4}$ & $X_{5}$ & $X_{6}$ \\
\hline
\end{tabular}

Table 2 Intrinsic sequence and evaluated sequences of simulation data sequences obeying normal distributions

\begin{tabular}{|c|c|c|c|c|c|c|}
\hline \multirow{2}{*}{ Number } & \multirow{2}{*}{$\begin{array}{c}\text { Intrinsic } \\
\text { sequence } \\
X_{1}\end{array}$} & \multicolumn{5}{|c|}{ Evaluated sequences } \\
\hline & & $X_{2}$ & $X_{3}$ & $X_{4}$ & $X_{5}$ & $X_{6}$ \\
\hline 1 & -0.01108 & 0.01760 & 0.00629 & 0.00351 & 0.01219 & -0.03287 \\
\hline 2 & 0.01453 & -0.00923 & 0.00522 & -0.01065 & -0.00306 & 0.01079 \\
\hline 3 & 0.00339 & -0.01048 & 0.00805 & -0.00759 & -0.01375 & -0.01116 \\
\hline 4 & 0.00250 & -0.00047 & -0.00922 & -0.00029 & -0.00590 & -0.00274 \\
\hline 5 & -0.00514 & 0.00213 & -0.01059 & -0.00177 & 0.00650 & 0.00604 \\
\hline 6 & -0.00953 & -0.01370 & -0.00805 & 0.02306 & 0.00525 & 0.00921 \\
\hline 7 & -0.00067 & 0.00705 & -0.01722 & -0.00896 & -0.02924 & -0.01726 \\
\hline 8 & 0.00844 & 0.00606 & 0.01351 & -0.00167 & 0.02062 & -0.00884 \\
\hline 9 & 0.01380 & 0.00096 & 0.02054 & 0.00594 & -0.01319 & 0.00445 \\
\hline 10 & -0.00059 & -0.00387 & -0.00093 & 0.03469 & 0.03177 & 0.01811 \\
\hline
\end{tabular}

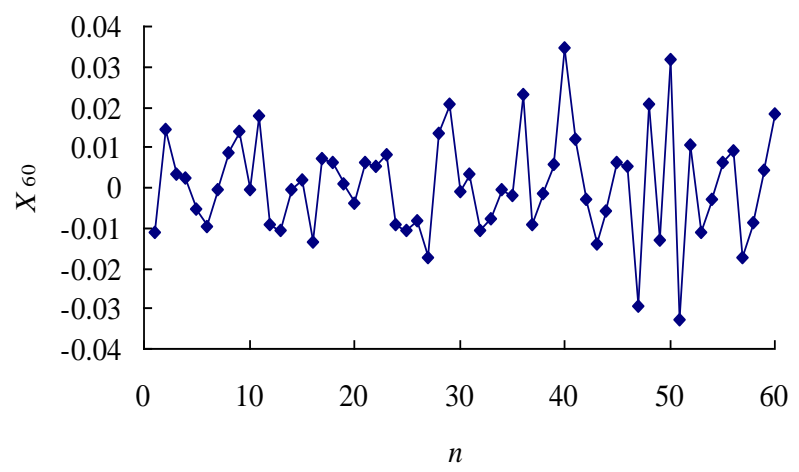

Figure 1. Simulation data sequence $X_{60}$ obeying normal distribution

Based on the fuzzy set theory, the intrinsic sequence and the evaluated sequences of the simulation data sequences obeying normal distributions are processed using the fuzzy norm method under the confidence level $P=95.44 \%$.

According to the evaluation method[7], Let $L=3$, and adjust the level $\lambda=\lambda^{*}$ to meet that the confidence level $P=95.44 \%$. It is estimated that the optimal level $\lambda^{*}$ and the optimal fuzzy uncertainty $U_{\lambda^{*}}$ at the optimal level $\lambda^{*}$. Then it is estimated that the relative error $\mathrm{d} U_{j \lambda^{*}}$ of the optimal fuzzy uncertainty $U_{j \lambda^{*}}$ between the evaluated sequences and the intrinsic sequence, and the 
estimated results as shown in Table 3.

Table 3 The estimated results of simulation data obeying normal distribution

\begin{tabular}{ccccccc}
\hline & Intrinsic & \multicolumn{5}{c}{ Evaluated sequences } \\
\cline { 3 - 7 } The estimated results & sequence & \multirow{2}{*}{$X_{2}$} & $X_{3}$ & $X_{4}$ & $X_{5}$ & $X_{6}$ \\
\hline The optimal level $\lambda^{*}$ & 0.3346 & 0.4555 & 0.2615 & 0.2501 & 0.352 & 0.343 \\
The fuzzy uncertainty $U_{j \lambda^{*}}$ & 0.039134 & 0.043335 & 0.047323 & 0.053436 & 0.055905 & 0.05746 \\
The relative error $\mathrm{d} U_{j \lambda^{*}}$ & & $10.735 \%$ & $20.926 \%$ & $36.546 \%$ & $42.855 \%$ & $46.829 \%$ \\
\hline
\end{tabular}

In theory, under the confidence level $P=95.44 \%$ corresponding to the fluctuation range of $( \pm 2 \sigma)$, the biggest relative error of the uncertainty of the machine tool is regarded as that $\mathrm{d} U=\left(6 \sigma_{1}-4 \sigma_{1}\right) / 4 \sigma_{1}=50 \%$.

From the estimated results in Table 3, under the confidence level $P=95.44 \%$, the relative error of the optimal fuzzy uncertainty is $\mathrm{d} U_{\lambda *}=45 \%<50 \%$, which can be considered as the critical value whether the machine tool is reliable or not. If $\mathrm{d} U_{\lambda *} \leq 45 \%$, the machine tool is reliable in this stage and the production can be continued; if $\mathrm{d} U_{\lambda^{*}}>45 \%$, the machine tool is unreliable in this stage and the operators must stop production timely.

According to the estimated results in Table 3, it is further that the reliability degree of the machine tool can be divided into five grades, like I, II, III, IV, V, respectively, to accurately evaluate the reliability of the machine tool, as follows:

I indicates that if $\mathrm{d} U_{\lambda * \leq 15 \%}$ and the machine tool is the best reliable;

II indicates that if $15 \%<\mathrm{d} U_{\lambda *} \leq 25 \%$ and the machine tool is the more reliable;

III indicates that if $25 \%<\mathrm{d} U_{\lambda *} \leq 35 \%$ and the machine tool is generally reliable;

IV indicates that if $35 \%<\mathrm{d} U_{\lambda *} \leq 45 \%$ and the machine tool is the worse reliable;

$\mathrm{V}$ indicates that if $\mathrm{d} U_{\lambda^{*}}>45 \%$ and the machine tool is unreliable.

Due to complex structures and mass influence factors, the uncertainty of the machine tool is unknown. In the actual process, it is in consistent with the actual situation that the reliability of the machine tool appears better or worse than before.

Prediction experiment of evaluating the reliability of the machine tool. Based on the evaluation method[7], the experimental data of the outer raceway roundness for the tapered roller bearing with 30204 are collected to evaluate the reliability of the machine tool.

After a special grinder adjustment, 30 sets of the bearings are selected randomly in the normal production to measure their outer raceway roundness, and the measured data can constitute the experimental data sequence $X_{30}$ of the outer raceway roundness of the bearings, as shown in Fig. 2.

The former 10 measured data in $X_{30}$ are considered as the intrinsic sequence $X_{1}$ of outer raceway roundness and the later 20 measured data in $X_{30}$ are equally divided into two groups and each group of 10 measured data, which are considered as the evaluated sequences $X_{2}, X_{3}$, respectively, as shown in Table 4.

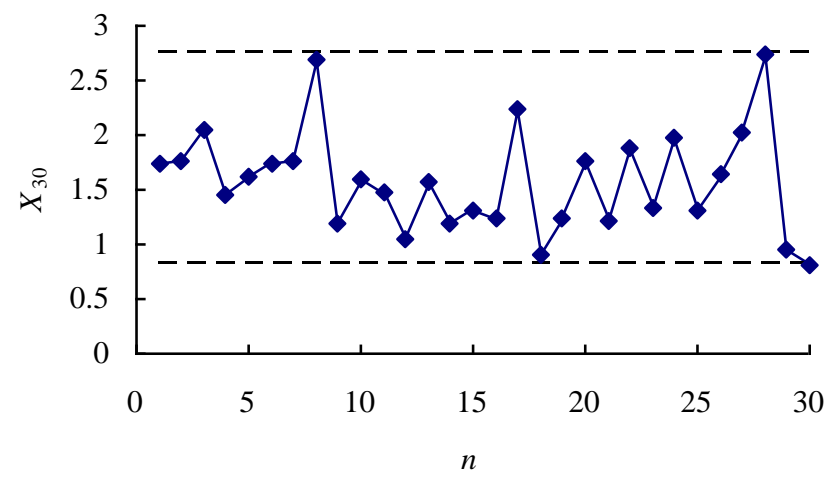

Figure 2. The experimental data sequence $X_{30}$ of outer raceway roundness of the bearings 
Table 4 Intrinsic sequence and evaluated sequences of outer raceway roundness $[\mu \mathrm{m}]$

\begin{tabular}{cccc}
\hline \multirow{2}{*}{ Number } & Intrinsic & \multicolumn{2}{c}{ Evaluated sequences } \\
\cline { 3 - 4 } sequence $X_{1}$ & $X_{2}$ & $X_{3}$ \\
\hline 1 & 1.74 & 1.47 & 1.21 \\
2 & 1.76 & 1.04 & 1.88 \\
3 & 2.04 & 1.56 & 1.34 \\
4 & 1.46 & 1.19 & 1.98 \\
5 & 1.62 & 1.32 & 1.30 \\
6 & 1.73 & 1.23 & 1.64 \\
7 & 1.76 & 2.23 & 2.03 \\
8 & 2.70 & 0.90 & 2.73 \\
9 & 1.19 & 1.24 & 0.95 \\
10 & 1.60 & 1.77 & 0.80 \\
\hline
\end{tabular}

Based on the fuzzy set theory, the intrinsic sequence and evaluated sequences of the 30 experimental data of the outer raceway roundness of the bearings are processed by the fuzzy norm method under the confidence level $P=95.44 \%$.

According to the evaluation method[7], Let $L=3$, and adjust the level $\lambda=\lambda^{*}$ to satisfy that the confidence level $P=95.44 \%$. It is estimated that the optimal level $\lambda^{*}$ and the optimal fuzzy uncertainty $U_{\lambda *}$ at the optimal level $\lambda^{*}$. Then it is estimated that the relative error $\mathrm{d} U_{j \lambda *}$ of the optimal fuzzy uncertainty $U_{j \lambda *}$ between the evaluated sequences and the intrinsic sequence, and the estimated results as shown in Table 5.

Table 5 The estimated results of the measured data of the outer raceway roundness

\begin{tabular}{cccc}
\hline \multirow{2}{*}{ The estimated results } & Intrinsic & \multicolumn{2}{c}{ Evaluated sequences } \\
\cline { 3 - 4 } & sequence $X_{1}$ & $X_{2}$ & $X_{3}$ \\
\hline The optimal level $\lambda^{*}$ & 0.2276 & 0.2562 & 0.396 \\
The fuzzy uncertainty $U_{j \lambda *}$ & 2.050973 & 1.765948 & 2.575556 \\
The relative error $\mathrm{d} U_{j \lambda \lambda^{*}}$ & & $-13.897 \%$ & $25.5773 \%$ \\
\hline
\end{tabular}

In Fig. 2 and Table 4, it can be seen that the fluctuation range of the evaluated sequences $X_{3}$ is larger than the fluctuation range of the intrinsic sequence $X_{1}$, and the fluctuation range of the evaluated sequences $X_{2}$ is smaller than the intrinsic sequence $X_{1}$ for the 30 experimental data. The above descriptions are the characteristics of the 30 experimental data in themselves.

In Table 5, the relative error $\mathrm{d} U_{\lambda^{*}}$ is computed that $\mathrm{d} U_{2 \lambda^{*}}=-13.897 \%<0$, and it can indicate that the fluctuation range of the outer raceway roundness data of the bearings grows small and the reliability of the machine tool turns into better than before, which is the best reliable in I stage. The relative error $\mathrm{d} U_{3 \lambda *}$ is computed that $25 \%<\mathrm{d} U_{3 \lambda *}=25.5773 \%<35 \%$, it can indicate that the fluctuation range of the outer raceway roundness data of the bearings grows large and the larger fluctuation range still is in the permitted range of products. It is considered that the reliability of the machine tool is in III stage, which the machine tool is generally reliable in this stage.

Therefore, the prediction results for reliability of the machine tool by product performance data accord with the characteristic law of the experimental data. The reliability of the machine tool is accurately evaluated by the fuzzy set theory. Prediction experiments for reliability of the machine tool by product performance data have a certain reference value in the mechanical research fields.

\section{Summary}

Prediction experiment of judging the reliability of the machine tool shows that If $\mathrm{d} U_{\lambda *} \leq 45 \%$, the machine tool is reliable and the production can be continued; if $\mathrm{d} U_{\lambda^{*}}>45 \%$, the machine tool is unreliable and the production should timely be stopped and the inspection and maintenance for the machine tool should be implemented as soon as possible. It is further obtained the reliability degree 
of the machine tool can be divided into I, II, III, IV, V, respectively to accurately evaluate the reliability of the machine tool, which is a good basis for evaluating accurately the reliability of the machine tool.

Prediction experiments of evaluating the reliability of the machine tool show that the reliability of the machine tool is accurately evaluated by the fuzzy set theory.

\section{Acknowledgements}

This project is supported by National Natural Science Foundation of China (Grant Nos. 51475144, 50375011 and 50675011).

\section{References}

[1] M. Colledani, T.Tolio, A. Fischer, et al, Design and management of manufacturing systems for production quality, J. CIRP Annals-Manufacturing Technology. 63 (2014) 773-796.

[2] A. Hees, G. Reinhart, Approach for Production Planning in Reconfigurable Manufacturing Systems, J. Procedia CIRP. 33 (2015) 70-75.

[3] M. Lazzaroni, L. Cristaldi, L. Peretto, et al, Reliability Engineering, Springer, Berlin, 2011.

[4] T. Karaulova, M. Kostina, J. Sahno, Framework of reliability estimation for manufacturing processes, J. Mechanika. 18 (2012) 713-720.

[5] Y.J Zhang, L.L. Ge, Reliability analysis of machining systems by considering system cost, J. International Journal of Computer Integrated Manufacturing. 28 (2015) 836-843.

[6] A.P. Kuznetsov, Probability methods of evaluation and control of precision reliability of metal-cutting machine tools under thermal effects, J. Journal of Machinery Manufacture and Reliability. 44 (2015) 363-371.

[7] X.T. Xia, W. H. Zhu, B. Liu, Evaluation method for Stability of Manufacturing Process Based on Fuzzy Norm Method.

[8] M. Francesco, Applications of Fuzzy Sets Theory, Springer, Berlin, 2007.

[9] M. Izadikhah, A fuzzy goal programming based procedure for Machine Tool Selection, J. Journal of Intelligent and Fuzzy Systems. 28 (2015) 361-372.

[10]X.C. Sun, X.T. Xia, Y.B. Liu, Evaluation of Rolling Bearing Vibration Using Fuzzy Set Theory and Chaos Theory, J. Advanced Materials Research. 424-425 (2012) 338-341. 